\section{Equivalent-Circuit Analysis of a Broadband Printed Dipole With Adjusted Integrated Balun and an Array for Base Station Applications}

\author{
RongLin Li, Terence Wu, Bo Pan, Kyutae Lim, Joy Laskar, and
} Manos M. Tentzeris

\begin{abstract}
A printed dipole with an integrated balun features a broad operating bandwidth. The feed point of conventional balun structures is fixed at the top of the integrated balun, which makes it difficult to match to a $50-\Omega$ feed. In this communication, we demonstrate that it is possible to directly match with the $50-\Omega$ feed by adjusting the position of the feed point of the integrated balun. The printed dipole with the hereby presented adjustable integrated balun maintains the broadband performance and exhibits flexibility for the matching to different impedance values, which is extremely important for the design of antenna arrays since the mutual coupling between antenna elements commonly changes the input impedance of each single element. An equivalent-circuit analysis is presented for the understanding of the mechanism of the impedance match. An eight-element linear antenna array is designed as a benchmarking topology for broadband wireless base stations.
\end{abstract}

Index Terms-Antenna array, base station antenna, broadband antenna, integrated balun, printed dipole, wireless communications.

\section{INTRODUCTION}

It is well known that a dipole needs a balanced feed, such as a $\lambda / 4$ coaxial balun (see Fig. 1(a), $\lambda=$ the operating wavelength of the dipole) [1]. The coaxial balun consists of a coaxial line and a $\lambda / 4$ short-circuited wire. The outer conductor of the coaxial line and the $\lambda / 4$ short-circuited wire form a $\lambda / 4$ two-conductor shorted stub and present infinite impedance at the feed point which is fixed at the top of the balun, thus eliminating current flow on the outer surface of the outer conductor of the coaxial line. For a printed dipole, the coaxial balun can be replaced with an integrated balun (see Fig. 1(b) which is composed of a microstrip line, a $\lambda_{m g} / 4$ open stub $\left(\lambda_{m g}=\right.$ the guided wavelength of the microstrip line) and a $\lambda_{s g} / 4$ short-circuited slot line $\left(\lambda_{s g}=\right.$ the guided wavelength of the slot line). The strip conductor and the ground plane of the microstrip line are equivalent to the inner conductor and the outer conductor of the coaxial line, respectively. The slot line is equivalent to the two-conductor line in Fig. 1(a). The $\lambda_{m g} / 4$ open stub acts as a shorting circuit at the feed point. The printed dipole with the integrated balun features a broadband performance [2] $(\sim 40 \%)$ and has found applications in wireless communications [3] and antenna arrays [4]. However, the printed dipole with an integrated balun whose feed point is fixed at its top (called the fixed integrated balun) has to be designed at the dipole's resonant resistance of $\sim 80 \Omega$, hence requiring a $63-\Omega$ quarter wavelength transformer to match with $50-\Omega$

Manuscript received December 21, 2007; revised September 26, 2008. First published May 02, 2009; current version published July 09, 2009.

R.L. Li was with the School of Electrical and Computer Engineering, Georgia Institute of Technology, Atlanta, GA 30308 USA. He is now with the School of Electronic and Information Engineering, South China University of Technology, Guangzhou 510641, China (e-mail: lirl@ scut.edu.cn).

T. Wu, B. Pan, K. Lim, J. Laskar, and M. M. Tentzeris are with the School of Electrical and Computer Engineering, Georgia Institute of Technology, Atlanta, GA 30308 USA (e-mail: etentze@ece.gatech.edu).

Color versions of one or more of the figures in this communication are available online at http://ieeexplore.ieee.org.

Digital Object Identifier 10.1109/TAP.2009.2021967

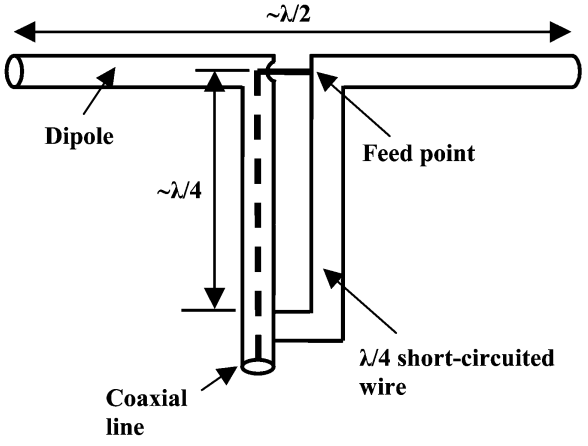

(a) Dipole with a $\lambda / 4$ coaxial balun.

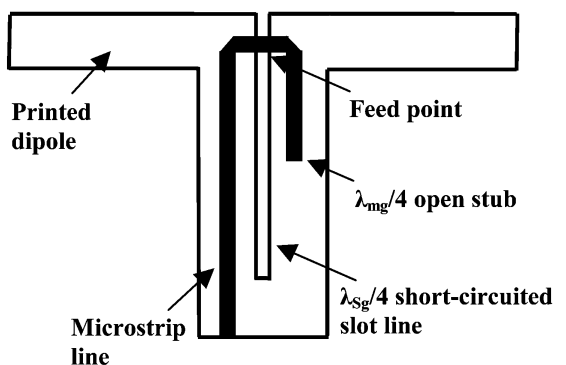

(b) Printed dipole with integrated balun.

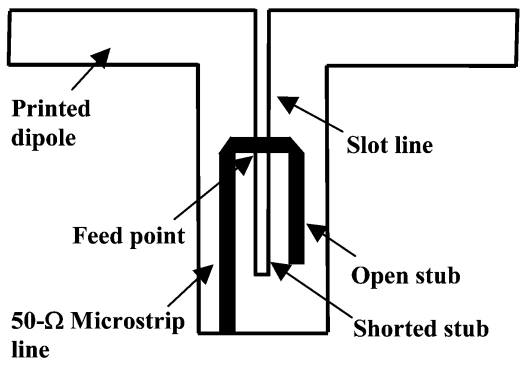

(c) Printed dipole with a modified integrated balun.

Fig. 1. Evolution of a printed dipole with integrated balun adjusted for match to a $50-\Omega$ feed. (a) Dipole with a $\lambda / 4$ coaxial balun. (b) Printed dipole with integrated balun. (c) Printed dipole with a modified integrated balun.

test equipment [2]. On the other hand, the bandwidth of the printed dipole with fixed integrated balun may be reduced by $30 \%-50 \%$ if it is directly connected to a $50-\Omega$ feed [3], [5]. In order to avoid both the use of a quarter wavelength transformer and the bandwidth reduction, the configuration of the printed dipole with fixed integrated balun has to undergo a simple-to-implement modification. It has been found that a printed Yagi-Uda dipole [6] or a T-dipole [7] can directly match to a $50-\Omega$ feed and achieve a broadband performance. But the printed Yagi-Uda dipole with integrated balun needs a high dielectric constant substrate for surface wave enhancement while the T-dipole has a high profile $(\sim 0.43 \lambda)$.

In this communication, we will demonstrate that the printed dipole with integrated balun can be matched to a $50-\Omega$ feed simply through an adjustment of the feed point of the integrated balun. By sliding down the feed point, the $\lambda_{s g} / 4$ short-circuited slot line is divided into a slot line and a shorted stub (see Fig. 1(c), which form a single-stub tuning circuit with a shunt shorted stub and transform a high impedance into a low impedance. Because the position of the feed point is an adjustable parameter, the adjusted integrated balun may match to different impedance values, which is useful for antenna arrays because the mutual coupling between array elements 


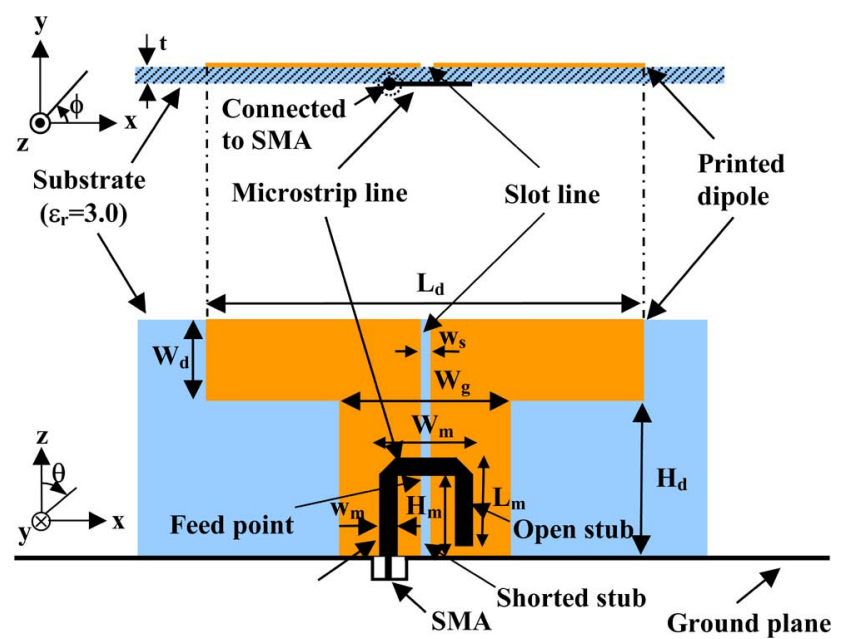

Fig. 2. Geometry of a printed dipole with adjusted integrated balun.

TABLE I

GeOMetric Parameters OF THE PRINTED Dipole With AdJusted INTEGRATED BALUN

\begin{tabular}{|c|c|c|c|}
\hline $\mathrm{L}_{\mathrm{d}}$ & $76 \mathrm{~mm}$ & $\mathrm{~W}_{\mathrm{m}}$ & $16 \mathrm{~mm}$ \\
\hline $\mathrm{W}_{\mathrm{d}}$ & $15 \mathrm{~mm}$ & $\mathrm{H}_{\mathrm{m}}$ & $15 \mathrm{~mm}$ \\
\hline $\mathrm{H}_{\mathrm{d}}$ & $27 \mathrm{~mm}$ & $\mathrm{~W}_{\mathrm{s}}$ & $1.0 \mathrm{~mm}$ \\
\hline $\mathrm{W}_{\mathrm{g}}$ & $30 \mathrm{~mm}$ & $\mathrm{~W}_{\mathrm{m}}$ & $2.5 \mathrm{~mm}$ \\
\hline $\mathrm{L}_{\mathrm{m}}$ & $15.5 \mathrm{~mm}$ & $\mathrm{t}$ & $1.0 \mathrm{~mm}$ \\
\hline
\end{tabular}

may change the input impedance of each antenna element. An equivalent-circuit analysis is presented in Section II to understand the impedance matching procedure. An eight-element linear antenna array is designed in Section III as a benchmarking topology for wireless base station applications.

\section{ANTENNA ElEMENT AND EQUIVALENT-CIRCUIT ANALYSIS}

Consider a printed dipole with an adjusted integrated balun as illustrated in Fig. 2, which has been fabricated on a substrate of RO4230, a Rogers Corporation's high frequency circuit material with a dielectric constant $\left(\varepsilon_{r}\right)$ of 3.0 and a loss tangent of 0.002 . The thickness $(t)$ of the substrate is $1.0 \mathrm{~mm}$. The integrated balun consists of a microstrip line and a slot line which are printed on the front side and the back side of the substrate, respectively; the dipole is printed on the backside as well. This structure is designed for wireless base station applications at the $2-\mathrm{GHz}$ band, which covers the frequency range from $1.7-2.5 \mathrm{GHz}$ (its center frequency $=2.1 \mathrm{GHz}$ ). The printed dipole with adjusted integrated balun is mounted (at center) above a ground plane with dimensions of $200 \mathrm{~mm}$ (in the $x$ direction) $\times 160 \mathrm{~mm}$ (in the $\mathrm{y}$ direction) and fed by an SMA connector underneath the ground plane. To keep the antenna profile as low as possible, the slot line is short-circuited directly by the ground plane. As a result, the height of the antenna (from the center of the printed dipole to the ground) is roughly a quarter wavelength of the slotline. The geometric parameters of the antenna are listed in Table I.

To understand the mechanism of impedance matching, the printed dipole with adjusted integrated balun is analyzed using an equivalentcircuit model shown in Fig. 3. The equivalent-circuit model has been used to analyze various antenna structures, such as a tapered dipole [8] or a bow-tie dipole [9]. Here the printed dipole can be considered to be fed by a slot line which is coupled to a microstrip line on the one hand and extended to a shorted stub on the other hand. The center-fed input impedance of the printed dipole alone (without the integrated

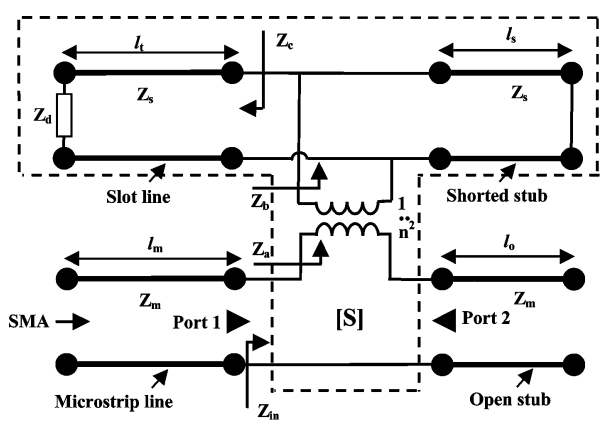

Fig. 3. Equivalent circuit of the printed dipole with adjusted integrated balun $\left(l_{m}=H_{m}+W_{m} / 2, l_{o}=L_{m}+W_{m} / 2-w_{m}, l_{t}=H_{d}+W_{d} / 2-H_{m}-\right.$ $\left.w_{m} / 2, l_{s}=H_{m}+w_{m} / 2\right)$.

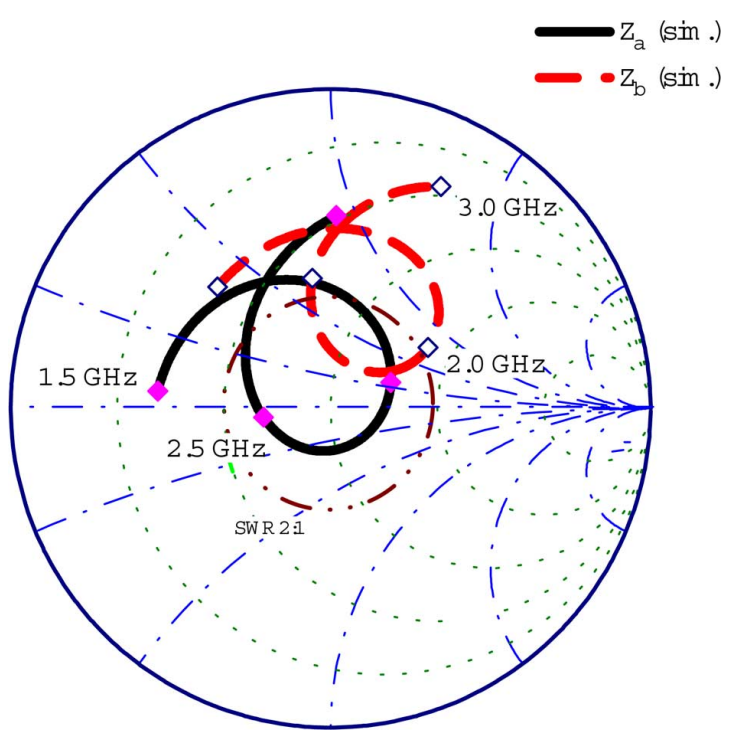

Fig. 4. Impedances $Z_{a}$ and $Z_{b}$ for calculation of the impedance transformer coefficient $\left(n^{2}\right) . Z_{a}$ is calculated using the $\mathrm{S}$ parameters of a two-port network as illustrated in the inset. $Z_{b}$ is obtained by directly driving the printed dipole at the adjusted feed point with a voltage source.

balun) is denoted by $Z_{d}$. The impedance looking into the slot line at the feed point is $Z_{c}$, which can be obtained through the transmission line impedance equation

$$
Z_{c}=Z_{0 s} \frac{Z_{d}+j Z_{0 s} \tan \beta_{s} l_{t}}{Z_{0 s}+j Z_{d} \tan \beta_{s} l_{t}}
$$

where $Z_{0 s}, \beta_{s}$, and $l_{t}$ are the characteristic impedance, phase constant, and the length of the slot line, respectively. The slot line parameters, $Z_{0 s}$ and $\beta_{s}$, can be calculated using the empirical formulas presented in [10]. A parallel combination of the impedance $Z_{c}$ with the shorted stub leads to an impedance $Z_{b}$

$$
Z_{b}=\frac{j Z_{c} Z_{0 s} \tan \beta_{s} l_{s}}{Z_{c}+j Z_{0 s} \tan \beta_{s} l_{s}}
$$

where $l_{s}$ is the length of the shorted stub.

The coupling between the slot line and the microstrip line can be represented as an ideal transformer which has a turn ratio of $n$. Following the transformer, the impedance $Z_{b}$ is transformed into $Z_{a}$

$$
Z_{a}=n^{2} Z_{b}
$$




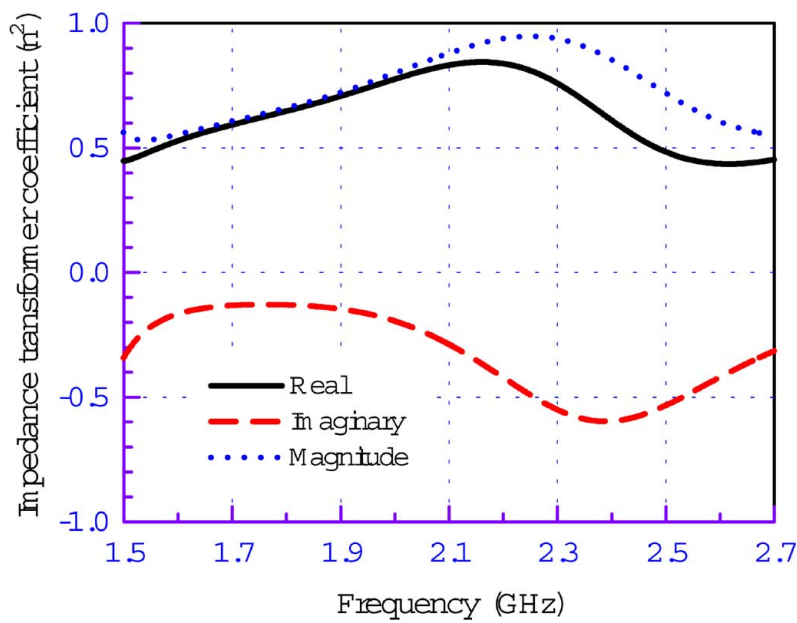

Fig. 5. Impedance transformer coefficient $\left(n^{2}\right)$ in the equivalent circuit calculated from $n^{2}=Z_{b} / Z_{a}$.

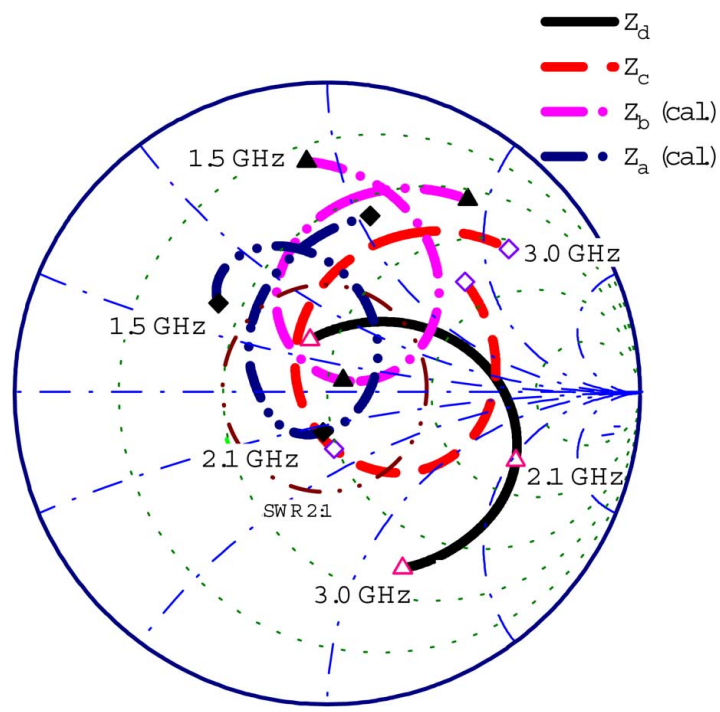

Fig. 6. Impedance matching procedure of the printed dipole with adjusted integrated balun starting from $Z_{d}$ which is obtained by directly feeding the printed dipole alone at its center with a voltage source. $Z_{a}, Z_{b}$, and $Z_{c}$ are calculated by (1), (2), and (3), respectively.

where $n^{2}$ is called the impedance transformer coefficient. Finally, the input impedance $\left(Z_{i n}\right)$ looking from the microstrip line at the feed point is obtained by taking into account the open stub, which gives

$$
Z_{\text {in }}=Z_{a}-j Z_{0 m} \cot \beta_{m} l_{o}
$$

where $Z_{0 m}, \beta_{m}$, and $l_{o}$ are the characteristic impedance, phase constant, and the length of the open stub, respectively.

To calculate the input impedance $Z_{i n}$, it first needs to determine the impedance transformer coefficient $n^{2}$ which is difficult to obtain through an analytical method. Therefore, we determined it by numerical simulation which was carried out using CST MicroStripes 7.5. First we obtained $Z_{b}$ by directly feeding the dipole with the slot line (but without the microstrip line) at the feed point with a voltage source.

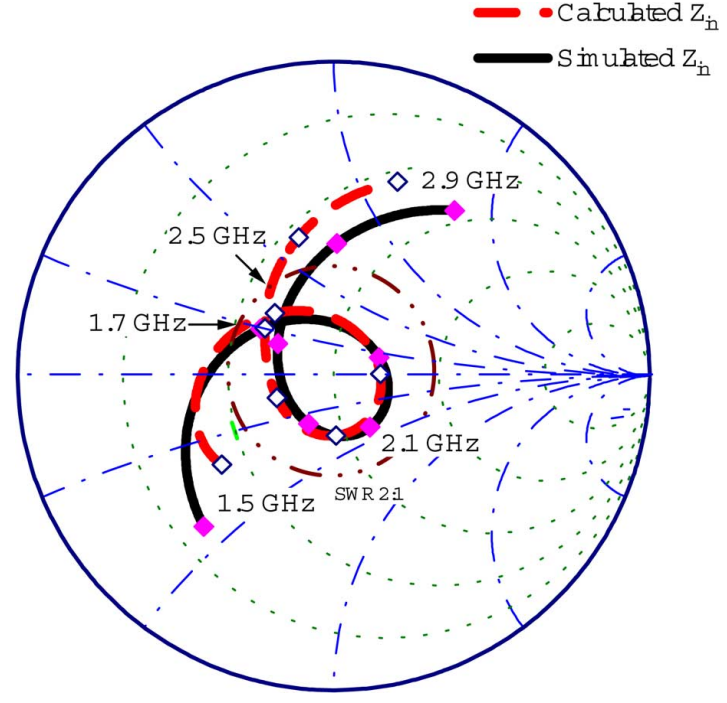

Fig. 7. Comparison between calculated (by (4)) and simulated input impedances of the printed dipole with adjusted integrated balun.

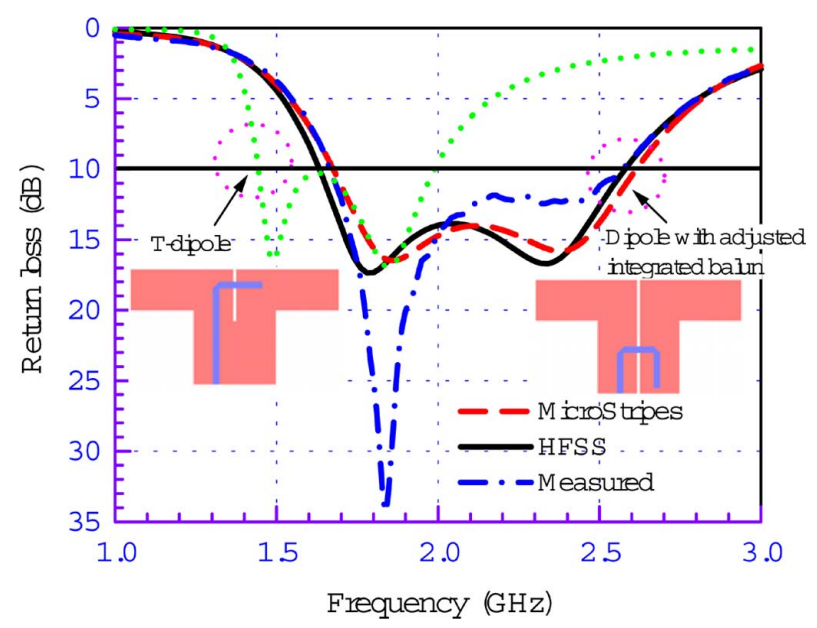

Fig. 8. Simulated and measured results for return loss of the printed dipole with adjusted integrated balun and comparison with a T-dipole.

Then $Z_{a}$ was determined from the $\mathrm{S}$ parameters of the two-port network enclosed in Fig. 3, which can be expressed as [11]

$$
Z_{a}=Z_{0 m} \frac{\left(1+S_{11}\right)\left(1+S_{22}\right)-S_{12} S_{21}}{2 S_{21}} .
$$

The simulated results for $Z_{a}$ and $Z_{b}$ are given in a Smith chart of Fig. 4. Upon the determination of $Z_{a}$ and $Z_{b}, n^{2}$ is calculated from (3). The result for $n^{2}$ is plotted in Fig. 5, which shows a negative imaginary part and a magnitude of less than one. This means that the coupling is capacitive and the transformer steps down the impedance.

Now let's examine the procedure of the impedance match. We start with the input impedance $\left(Z_{d}\right)$ of the printed dipole without the integrated balun (i.e., remove the slot line and the microstrip line). $Z_{d}$ was obtained by simulation with of a voltage source excitation at its center. The result for $Z_{d}$ is plotted in Fig. 6. We can see that $Z_{d}$ has a high impedance of $(144-j 105) \Omega$ at the center frequency. Note that printed dipole has an anti-resonant (i.e., parallel-resonant) frequency of $\sim 1.9 \mathrm{GHz}$ and a anti-resonant resistance of $\sim 180 \Omega$. Following the slot line, we calculate $Z_{c}$ using (1), which leads to a 


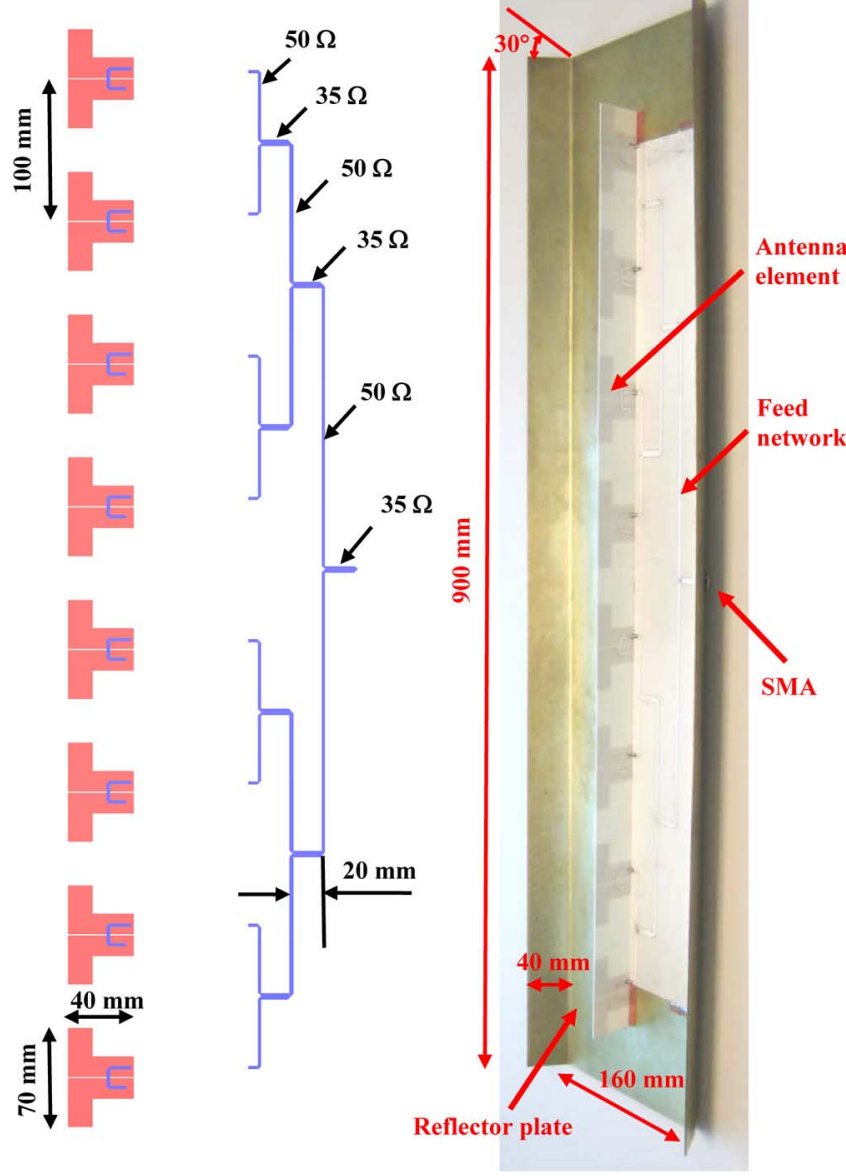

Fig. 9. Eight-element linear array of the printed dipole with adjusted integrated balun. (a) Antenna elements. (b) Feed network. (c) Prototype.

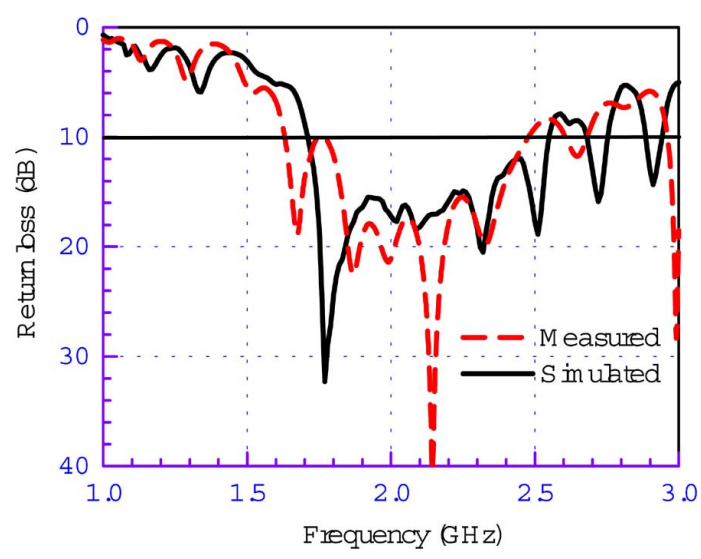

Fig. 10. Simulated and measured results for return loss of the eight-element linear array antenna.

lower impedance of $(48-j 18) \Omega$. This means that the slot line can transform a high impedance into a low impedance. To transform the high impedance $Z_{d}$ into an impedance close to $\sim 50 \Omega$ in real part, the characteristic impedance of the slot line should be $\sim 110 \Omega$. The locus of $Z_{d}$ is rotated clockwise in the Smith chart to reach $Z_{c}$. After a parallel combination with a shorted stub, we obtain $Z_{b}$ from (2). Because the shorted stub is shorter $(\sim \pi / 4)$ than a quarter guided wavelength, the resultant $Z_{b}$ becomes more inductive and an impedance loop appears. With the help of the capacitive coupling (i.e., the transformer) between the slot line and

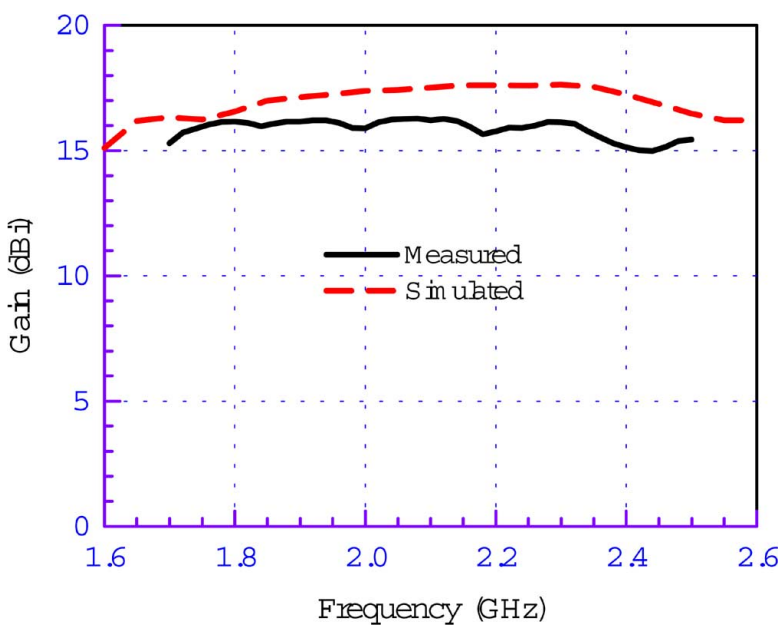

Fig. 11. Simulated and measured gains of the eight-element linear array antenna.
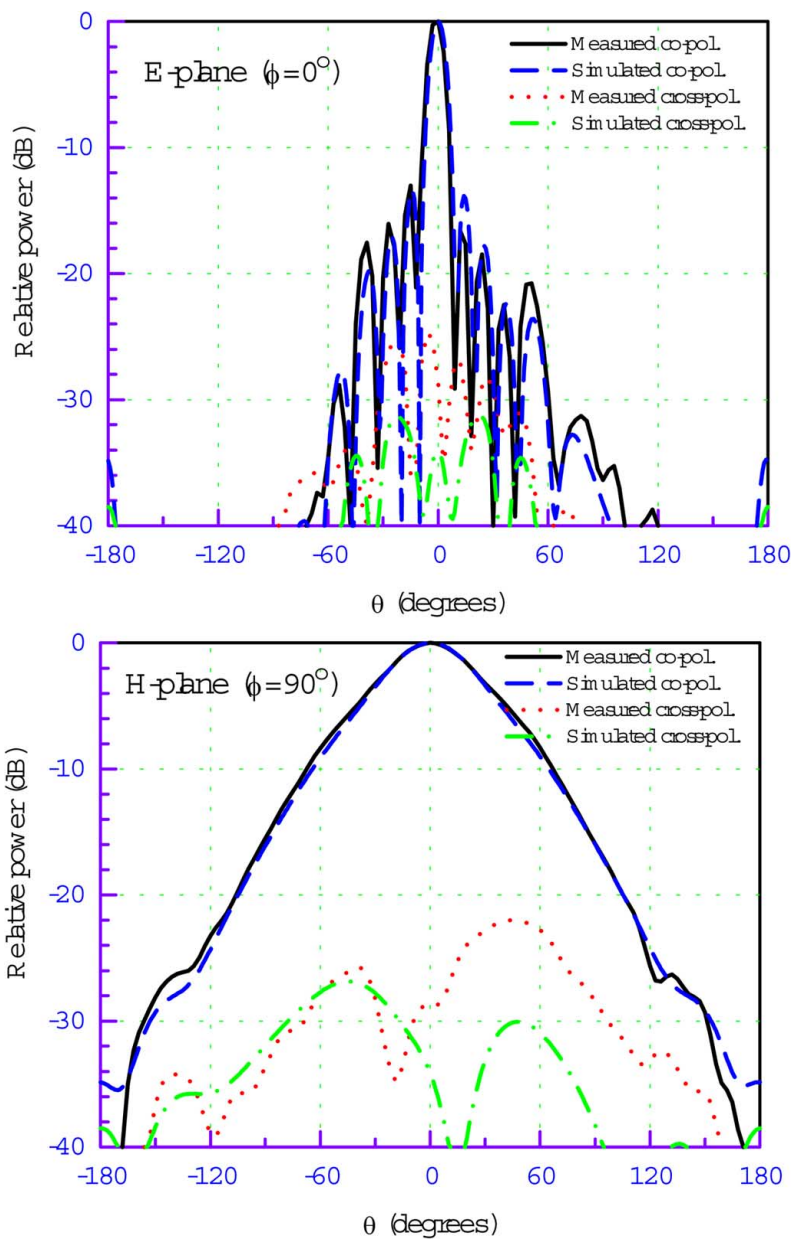

Fig. 12. Radiation patterns of the eight-element linear array antenna simulated and measured at $2.1 \mathrm{GHz}$.

the microstrip line, we get $Z_{a}$ which shows a less inductive component than $Z_{b}$. Note that the impedance loop of $Z_{a}$ is not completely enclosed by the SWR $=2: 1$ circle. To move the impedance loop of $Z_{a}$ into the SWR $=2: 1$ circle, an open stub is involved. The open stub has a length of one quarter wavelength around the center frequency. But it not only serves as a shorted circuit, but also acts 
as a varying reactance which cancels the inductive component of $Z_{a}$ in the lower frequency. We have calculated $Z_{i n}$ using (4) and compared with simulation results in Fig. 7. It can be seen that the calculated and simulated results are in good agreement and the impedance loops are located within the SWR $=2: 1$ circle, which means a good impedance match over a broad bandwidth. The good agreement validates the equivalent-circuit analysis. A slight difference is mainly due to three reasons: 1) we have neglected the equivalent lengths associated with the nonzero inductance at the shorted stub and the nonzero capacitance at the open stub; 2) the radiation from the slot line and the microstrip line was not taken account; 3) the attachment of the slot line to the printed dipole may change its input impedance $\left(Z_{d}\right)$.

The simulation result using the MicroStripes for return loss of the printed dipole with adjusted integrated balun was crosschecked using HFSS and is compared with experimental result in Fig. 8. Good agreement is observed and the bandwidth for $\mathrm{RL} \geq 10 \mathrm{~dB}$ is about $41 \%$, covering the frequency band $1.7-2.5 \mathrm{GHz}$. To demonstrate the advantage of the printed dipole with adjusted integrated balun, we have also plotted the return loss in the figure for a T-dipole which has the same overall dimensions $(76 \mathrm{~mm} \times 42 \mathrm{~mm})$. It is seen that the bandwidth of the T-dipole is $\sim 30 \%$, much less than the bandwidth $(\sim 40 \%)$ of the printed dipole with adjusted integrated balun. To enhance the bandwidth of the T dipole, it would be needed to increase the height of the T-dipole, which may lead to a reduced gain and a dipped radiation pattern (the maximum radiation is no longer at broadside) [12].

\section{Antenna ArRay for Base Station ApPlications}

To demonstrate the potential applications of the printed dipole with adjusted integrated balun, we have designed an eight-element linear array for wireless base station communications. The antenna elements were slightly modified to take into account the mutual coupling among these elements. It was found that the mutual coupling tends to shift down the operating frequency of the printed dipole from $1.7-2.5 \mathrm{GHz}$ to $1.5-2.3 \mathrm{GHz}$ and the impedance matching became worse at the lower frequency. The operating frequency shift down is reasonable because the neighboring elements of an antenna tend to effectively increase the size of the antenna. We scaled down the length and the overall height of the printed dipole from $76 \mathrm{~mm}$ to $70 \mathrm{~mm}$ for the length and $42 \mathrm{~mm}$ to $40 \mathrm{~mm}$ for the overall height. The position of the feed point was adjusted from $H_{m}=15 \mathrm{~mm}$ to $H_{m}=14 \mathrm{~mm}$ and the length of the open stub was shortened from $L_{m}=15.5 \mathrm{~mm}$ to $L_{m}=12.5 \mathrm{~mm}$. The arrangement of the antenna elements is shown in Fig. 9(a). The antenna array is fed by a corporate feed network as depicted in Fig. 9(b). The 50- $\Omega$ microstrip lines from neighboring elements are joined at a $\mathrm{T}$-junction and transformed through a $35-\Omega$ quarter-wave transformer back to a single $50-\Omega$ line. The antenna array and the feed network were fabricated on the same RO4230 board and cut out afterward. The antenna array is mounted on the center of a reflector plate with dimensions of $900 \mathrm{~mm} \times 160 \mathrm{~mm}$ while the feed network is placed on the plate and fed by an SMA connector from the backside of the reflector. The reflector plate has a pair of side walls to reduce the backside radiation. A prototype of the assembled antenna array is displayed in Fig. 9(c). The measured and simulated results for return loss of the antenna array are presented in Fig. 10 and shows reasonable agreement. The measured bandwidth is about $40 \%$ and covers the design frequency 1.7-2.5 GHz. Measured gain is compared with simulated result in Fig. 11. It is interesting to note that the measured gain has an almost constant value of $12 \mathrm{dBi}$ over the design frequency, useful for broadband wireless applications. The constant gain is due to the fact that the array factor increases with frequency while the gain of the antenna element decreases as frequency increases. The discrepancy between the simulated and measured gains is due to the calibration errors. (The gain and radiation pattern of the antenna array were measured using Satimo's Multiprobe Antenna Measurement System.) The radiation pattern measured at $2.1 \mathrm{GHz}$ is compared with simulation result in Fig. 12 and good agreement is obtained. The cross-polarized component is less than $20 \mathrm{~dB}$. The sidelobe level is less than $14 \mathrm{~dB}$ and the backside level is less than 35 $\mathrm{dB}$ due to the effect of the side walls. The antenna array should be found applications in base stations for wireless communications with the requirements for almost frequency-independent gain and broadband matching $(\sim 40 \%)$.

\section{CONCLUSION}

A printed dipole with an adjusted integrated balun is investigated with the help of an equivalent-circuit analysis. It is found that this topology can directly match to a $50-\Omega$ feed and has a bandwidth of more than $40 \%$. The broadband impedance matching can be achieved simply by adjusting the position of the feed point of the integrated balun. With the proposed adjustable integrated balun, an eight-element linear antenna array has been developed, which has an almost constant gain of $\sim 16 \mathrm{dBi}$ over the frequency range from 1.7 to $2.5 \mathrm{GHz}$ and thus may find applications in base stations for $2 \mathrm{G}, 3 \mathrm{G}$, and $4 \mathrm{G}$ wireless communications.

\section{ACKNOWLEDGMENT}

The authors would like to thank Satimo at Atlanta, the microwave version company, for providing the pattern measurement facility and Ms. K. Rutkowski for doing the measurement. The authors also wish to acknowledge the support of Georgia Electronic Design Center (GEDC).

\section{REFERENCES}

[1] C. A. Balanis, Antenna Theory: Analysis and Design. New York: Wiley, 2005, pp. 538-541.

[2] B. Edward and D. Rees, "A broadband printed dipole with integrated balun," Microw. J., pp. 339-344, May 1987.

[3] H.-R. Chuang and L.-C. Kuo, "3-D fdtd design analysis of a 2.4-GHz polarization-diversity printed dipole antenna with integrated balun and polarization-switching circuit for wlan and wireless communication applications," IEEE Trans. Microw. Theory Tech., vol. 51, pp. 374-381, Feb. 2003.

[4] J.-P. R. Bayard, "Analysis of infinite arrays of microstrip-fed dipoles printed on protruding dielectric substrates and covered with a dielectric radome," IEEE Trans. Antennas Propagat., vol. 42, pp. 82-89, Jan. 1994.

[5] D. Jaisson, "Fast design of a printed dipole antenna with an integrated balun," Proc. Inst. Elect. Eng. Microw. Antennas Propag., vol. 153, no. 4, pp. 398-394, Aug. 2006.

[6] K. M. K. H. Leong, Y. Qing, and T. Itoh, "Surface wave enhanced broadband planar antenna for wireless applications," IEEE Microw. Wireless Compo. Lett., vol. 11, pp. 62-64, Feb. 2001.

[7] C. Sabatier, "T-dipole arrays for mobile applications," IEEE Antennas Propag. Mag., vol. 45, no. 6, pp. 2-26, Dec. 2003.

[8] A. B. Smolders and M. J. Arts, "Wide-band antenna element with integrated balun," Proc. IEEE-APS, vol. 3, pp. 1394-1397, Jun. 1998.

[9] M. J. Arts and A. B. Smolders, "Design and construction of an array of vertically orient bow-tie antennas for a new generation of radio telescopes," in Proc. 29th Eur. Microw. Conf., 1999, pp. 259-262.

[10] R. Janaswamy and D. H. Schaubert, "Characteristic impedance of a wide slotline on low-permittivity substrates," IEEE Trans. Microw. Theory Tech., vol. 34, pp. 900-902, Aug. 1986.

[11] D. M. Pozar, Microwave Engineering, Second ed. New York: Wiley, 1998, pp. 211-211.

[12] Z. Zhou, S. Yang, and Z. Nie, "A novel broadband printed dipole antenna with low cross polarization," IEEE Trans. Antennas Propag., vol. 55, pp. 3091-3093, Nov. 2007. 